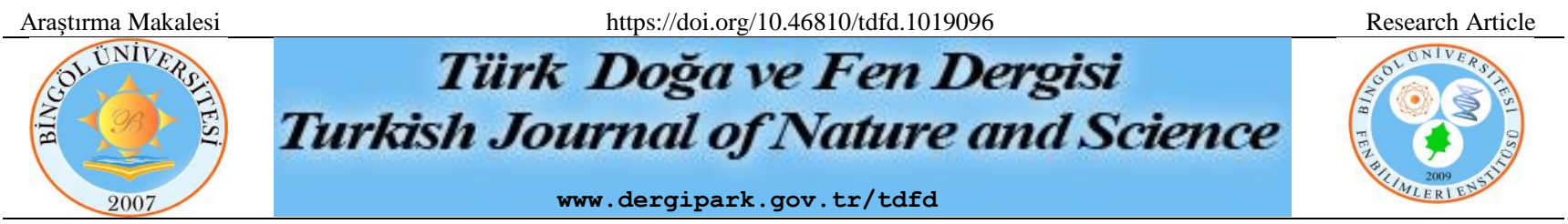

Probing Compressed Slepton Production at Future Large Hadron Collider

\author{
Ali ÇELİK ${ }^{1 *}$, Fatma Betül GÜZELOĞLU ${ }^{2}$ \\ ${ }^{1}$ Burdur Mehmet Akif Ersoy Üniversitesi, Fen-Edebiyat Fakültesi, Fizik Bölümü, Burdur, Türkiye \\ ${ }^{2}$ Burdur Mehmet Akif Ersoy Üniversitesi, Fen-Edebiyat Fakültesi, Fizik Bölümü, Burdur, Türkiye \\ Ali ÇELIKK ORCID No: 0000-0001-8218-6512 \\ Fatma Betül GÜZELOĞLU ORCID No: 0000-0002-0633-340X
}

*Corresponding author: ali.celik@cern.ch

(Alınış: 04.11.2021, Kabul: 20.11.2021, Online Yayınlanma: 31.12.2021)

\begin{abstract}
Keywords
SUSY,

LHC,

$33 \mathrm{TeV}$

Abstract: Various supersymmetry searches are carried out at the Large Hadron Collider (LHC) at CERN. While some searches have focused on the color sector, others focused on electroweak production of charginos-neutralinos and sleptons. Since no sign of supersymmetry has yet to be found, limits are set on the masses of the supersymmetric particles. In this phenomenological study, we probe direct slepton signal production in compressed mass spectra scenario at the potential future proton-proton collider operating at a center of mass energy of $33 \mathrm{TeV}$. We show that slepton signal with masses up to $270 \mathrm{GeV}$ can be discovered at the future hadron collider with the data corresponding to $100 \mathrm{fb}^{-1}$ integrated luminosity when $\Delta \mathrm{M}=5 \mathrm{GeV}$.
\end{abstract}

\title{
Büyük Hadron Çarpıştırıcısında Slepton Sinyalinin Küçük Kütle Farkı Durumunda İncelenmesi
}

Anahtar
Kelimeler
SÜSİ,
BHÇ,
$33 \mathrm{TeV}$

\begin{abstract}
Öz: CERN'deki Büyük Hadron Çarpıştırıcısı'ndaki (BHÇ) deneylerden toplanan verilerle süpersimetri izi aranmaktadır. Araştırmalardan bazıları renkli süpersimetrik parçacıklara odaklanırken, bazıları ise chargino-nötralino veya sleptonların elektrozayıf üretimine odaklanmışlardır. Şu ana kadar yapılan deneysel araştırmalarda süpersimetrik parçacık izine rastlanılmadığından, bu parçacıkların kütleleri üzerine sınırlamalar getirilmiştir. Bu fenomenolojik çalışmada, $33 \mathrm{TeV}$ 'lik Kütle Merkezi (K.M.) enerjisinde proton-proton çarpıştırıcısında slepton sinyali incelenmiş ve $100 \mathrm{fb}^{-1}$ 1şıklılığa karşılık gelen verilerle, en hafif süpersimetrik parçacık ile slepton arasındaki kütle farkının çok küçük olduğu durumda $(\Delta \mathrm{M}=5 \mathrm{GeV}), 270 \mathrm{GeV}$ 'ye kadar kütleye sahip slepton sinyalinin keşfedilebileceği gösterilmiştir.
\end{abstract}

\section{INTRODUCTION}

Standard Model (SM) of particle physics is one of the best theories that humankind has achieved so far. However, it is not considered a complete theory for a few reasons, some of which are that it is unable to answer the Higgs hierarchy problem and the existence of the dark matter whose presence is proved by the works [1-3]. However, the supersymmetric extension of the standard model (SUSY), predicting a superpartner for every corresponding particle in the SM, has the ability to solve the hierarchy problem in Higgs mass, and unify three interactions into a single one in addition to having a dark matter candidate.

Studies for searching supersymmetric particles are being carried out by CMS and ATLAS collaborations at the
LHC. Both experiments put limits on the masses of strongly produced colored supersymmetric particles, and their masses are excluded below $2 \mathrm{TeV}$ [4-12]. However, limits on the electroweakly produced charginos-neutralinos and sleptons are less constrained as these particles suffer from smaller production cross section in a hadron collider.

Directly produced sleptons are searched for by CMS and ATLAS experiments at both 8 and $13 \mathrm{TeV}$ [13-17] in the final states with di-leptons and the lightest supersymmetric particle (LSP). Since LSP leaves no trace in the detector, it contributes to missing energy $\left(E_{T}^{\text {miss }}\right)$ an important discriminator between SUSY signal and backgrounds in SUSY searches. Slepton signal production was also probed in phenomenology works in final states with $E_{T}^{\text {miss }}$, di-lepton and an initial state 
radiation (ISR jet) at $14 \mathrm{TeV}$ collision energy, assuming the mass difference between slepton and LSP are $\Delta \mathrm{M} \leq 20 \mathrm{GeV}$ and $\Delta \mathrm{M} \leq 60 \mathrm{GeV}[18,19]$. Ref. [18] was able to reach $5 \sigma$ discovery for left-handed sleptons of $\approx 150 \mathrm{GeV}$ with an integrated luminosity of $100 \mathrm{fb}^{-1}$. Using the search strategy of Ref. [19], it is shown that $4.7 \sigma$ with $300 \mathrm{fb}^{-1}$ data could be obtained for $m_{\widetilde{\mu}}=110$ $\mathrm{GeV}$ in the case of $\Delta \mathrm{M}=60 \mathrm{GeV}$. In another study on slepton production via vector boson fusion topology, $3-6 \sigma$ is obtained for slepton mass range $115-135 \mathrm{GeV}$ and mass splittings of 5-15 GeV [20].

We probe slepton pair production from the proton-proton (pp) collisions (depicted in Figure 1) at $33 \mathrm{TeV}$ at a potential future hadron collider called HE-LHC. Results are presented in the context of the R-parity conserving minimal supersymmetric extension of the SM (MSSM) $[21,22]$. We assume that neutralino $\left(\widetilde{\chi_{1}^{0}}\right)$ is LSP and purely Bino. In order to obtain correct relic density [23] LSP is assumed to be almost mass degenerate with slepton.

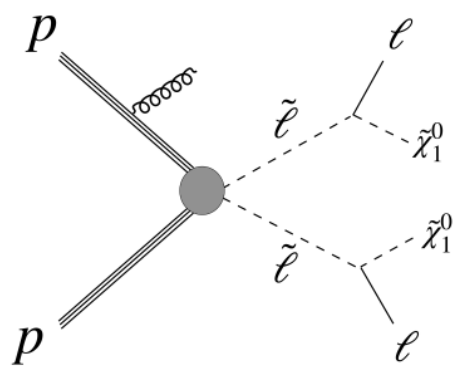

Figure 1. Slepton pair production along with an ISR jet coming from one of the incident partons.

\section{ANALYSIS STRATEGY}

The signal we probe in this analysis is the production of a pair of left/right-handed selectrons $(\tilde{e})$ or smuon $(\tilde{\mu})$ pair, which are produced from $Z^{*}$ or $\gamma^{*}$ exchange from quark anti-quark/gluon-quark pair, along with a single additional jet emitted from one of the incident partons. Left and right-handed selectrons and smuons are assummed to be mass degenerate. When the slepton pair is produced, both sleptons decays to LSP and the same flavor leptons, which are expected to be soft products in the case where the mass difference between slepton and LSP is small, called compressed mass spectra scenario. In the compressed scenario, we face the difficulty of reconstruction of leptons as they are most likely to be soft, and consequently, they are indistinguishable from the SM process $\bar{q} q \rightarrow \gamma^{*} / Z^{*} \rightarrow \bar{l} l$. In order to overcome the low acceptance due to reconstruction of the soft leptons, we require a highly energetic ISR jet, which will recoil against the sleptons and create a boost in transverse momentum $\left(p_{T}\right)$ of pair produced sleptons as well as in their decay products of same flavor opposite sign (SFOS) leptons and $\widetilde{\chi_{1}^{0}}$ pair. Hence, our signal is characterized by the existence of one hard jet, significant amount of missing energy, coming from LSPs, and SFOS lepton pair in the final state.
The major Standard Model backgrounds that have a similar topology as our signal are $t \bar{t}, V V+j e t$ and $Z+$ jets. Signals and all the SM backgrounds are generated with MadGraph_aMC@NLO version 2.6.7 [24] then pushed into Pythia 6 [25] for parton showering and hadronization, which is followed by detector simulation with Delphes 3 [26] using default delphes card prepared for HE-LHC [27]. While single vector backgrounds are simulated up to four partons, all other backgrounds and signal samples are generated up to two partons. Due to computational difficulties, some of the samples with higher production cross sections and higher jet multiplicity, such as $\bar{t} t$ and $Z+j e t s$ are generated $\approx 20$ and 30 million respectively. MLM scheme [28] is performed to avoid double counting. Parameter cards used in Madgraph for signal generation are produced with the SUSY spectrum generator called SUSY-HIT [29].

Results presented here are for the signal mass point of $m_{\tilde{l}}=235 \mathrm{GeV}$ with $\Delta \mathrm{M}=5 \mathrm{GeV}$; however, we scanned heavier slepton masses of $m_{\tilde{l}}=245,250,260$ and $270 \mathrm{GeV}$ for the cases of $\Delta \mathrm{M}=3,5 \mathrm{GeV}$. Event selection criteria that are global to all mass splittings are shown below. Residual cross sections after applying each cut for both SM backgrounds and the signal are shown in Table 1.

1. Veto on tagged hadronically decaying $\tau$

2. We require two SFOS leptons with $p_{T}>7 \mathrm{GeV}$ and $|\eta|<2.4$

3. Veto events with tagged b-jet with $p_{T}>30$ and $|\eta|<2.4 \mathrm{GeV}$ to reduce any contribution from $\mathrm{t} \overline{\mathrm{t}}$ background

4. Require only one hard jet with $p_{T}>180 \mathrm{GeV}$ and reject any events with additional jets having $p_{T}>$ $30 \mathrm{GeV}$.

5. Require $E_{T}^{\text {miss }}>180 \mathrm{GeV}$. Along with high jet $p_{T}$ requirement, this requirement will reduce significant amount of the remaining $t \bar{t}$ as well as $Z$ background.

6. Z-veto (i.e., rejects events with $81 \mathrm{GeV}<\mathrm{M}_{\mathrm{ll}}<$ $101 \mathrm{GeV}$ )

7. $\cos \theta_{l_{1}, l_{2}}^{*}<0.5$ applied. It is introduced in Ref. [30], applied in Ref. [31], and is effective to distinguish spin-0 parent particles from spin-1, which also yields the same final products as in slepton decay.

8. Upper cut on scalar sum of the $p_{T}$ of both leptons $\left(p_{T_{l 1}}+p_{T_{l 2}}<50 \mathrm{GeV}\right.$ for $\Delta \mathrm{M}=5 \mathrm{GeV}$, $p_{T_{l 1}}+p_{T_{l 2}}<35$ for $\left.\Delta \mathrm{M}=3 \mathrm{GeV}\right)$

9. As the requirement of a highly energetic jet creates a boost in slepton or, in other words in LSPs, decay products of sleptons, $E_{T}^{\text {miss }}$ and jet will be back-toback in compressed scenario. Thus, $\Delta \Phi\left(\right.$ Jet, $\left.E_{T}^{\text {miss }}\right)>0.95$ requirement will be beneficial to reduce backgrounds while keeping a large amount of the signal.

10. Along with $E_{T}^{\text {miss }}$, both leptons will recoil against ISR jet. Thus, a lower cut of 0.65 on $\phi$ difference between jet and vector sum of $1^{\text {st }}$ and $2^{\text {nd }}$ leptons' 
momentum ( $\Delta \Phi\left(\right.$ Jet, $\left.\mathrm{l}_{1} \mathrm{l}_{2}\right)>0.65$ ) will help to eliminate backgrounds as well.

11. Upper cut on mass difference between kinematic variable, stransverse mass $\mathrm{m}_{\mathrm{T} 2}$ [32,33] and trial mass, for given LSP mass. $\mathrm{m}_{\mathrm{T} 2}$ is used to estimate the minimum mass of the mother particle (slepton) from the kinematics of the visible and invisible decay products. In order to calculate the variable, we make use of the bisection code provided by the authors of reference [34]. Masses of each mother and daughter particles are assumed to be equal. The code requires transverse momentum of visible particles, missing transverse momentum, and masses of LSP's as input.

Table 1. Residual cross section (fb) for backgrounds and signal sample after applying each cut given 1-11.

\begin{tabular}{|c|c|c|c|c|c|c|}
\hline Selections & $\mathbf{t} \overline{\mathbf{t}}+\mathbf{j e t s}$ & $\mathrm{ZZ}+$ jets & WZ + jets & WW + jets & $\mathrm{Z}+$ jets & $S_{230}^{235}$ \\
\hline Initial & 3919650.00 & 40154.80 & 149999.00 & 308871.00 & 137857000.00 & 129.72 \\
\hline$\tau$ Veto & 3562280.00 & 38706.20 & 143452.00 & 296032.00 & 136270000.00 & 128.74 \\
\hline 2OSSF & 44919.70 & 1571.05 & 2767.32 & 2527.33 & 2462960.00 & 10.58 \\
\hline b-jet Veto & 13190.2 & 1423.38 & 2670.50 & 2497.93 & 2453960.00 & 10.46 \\
\hline Jet $\mathrm{p}_{\mathrm{T}}>180 \mathrm{GeV}$ & 65.14 & 8.89 & 20.69 & 43.63 & 3382.10 & 0.34 \\
\hline $\mathrm{MET}>180 \mathrm{GeV}$ & 21.35 & 1.75 & 2.25 & 14.68 & 47.50 & 0.33 \\
\hline $81<\mathrm{M}_{\mathrm{ll}}<101$ & 18.43 & 0.07 & 0.65 & 13.18 & 9.50 & 0.32 \\
\hline $\cos \theta_{\mathrm{l}_{1}, \mathrm{l}_{2}}^{*}<0.5$ & 10.40 & 0.05 & 0.41 & 8.93 & 9.50 & 0.26 \\
\hline $\mathrm{p}_{\mathrm{T}_{11}}+\mathrm{p}_{\mathrm{T}_{12}}<50$ & 0.55 & 0.01 & 0.02 & 0.93 & - & 0.23 \\
\hline$\Delta \Phi($ Jet, MET $)$ & - & 0.01 & 0.01 & 0.81 & - & 0.22 \\
\hline$\Delta \Phi($ Jet, l112) & - & 0.01 & 0.01 & 0.53 & - & 0.19 \\
\hline $\mathrm{m}_{\mathrm{T} 2}-\mu<5$ & - & - & - & 0.06 & - & 0.18 \\
\hline
\end{tabular}

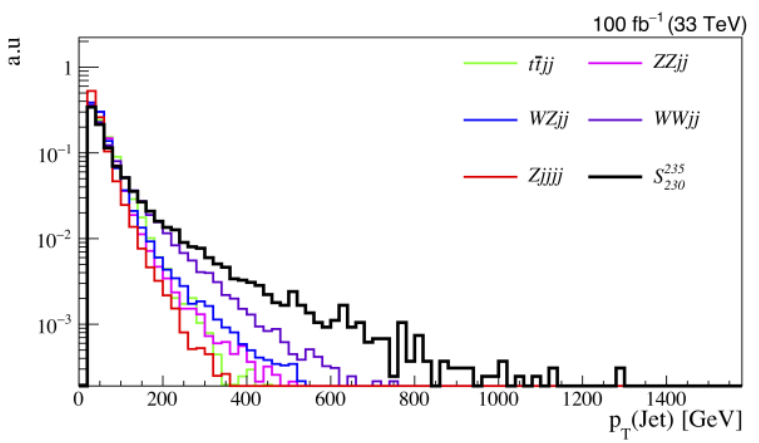

Figure 2. Leading jet $p_{T}$ distribution, normalized to unity. Events shown in the plot have passed cuts 1-3.

Figure 2 shows leading jet transverse momentum from both SM backgrounds and signal event, a $235 \mathrm{GeV} \tilde{l}$ decaying to a $230 \mathrm{GeV}$ LSP, after applying the first three event selection criteria. To get a transverse boost that will make the signal stand out against backgrounds, we require a lower cut of $180 \mathrm{GeV}$ on jet $p_{T}$. For the mass point $(235,230) \mathrm{GeV}$, jet $p_{T}$ cut removes more than $99.8 \%$ of the backgrounds and increases the signal to background ratio by a factor of $\approx 23$.

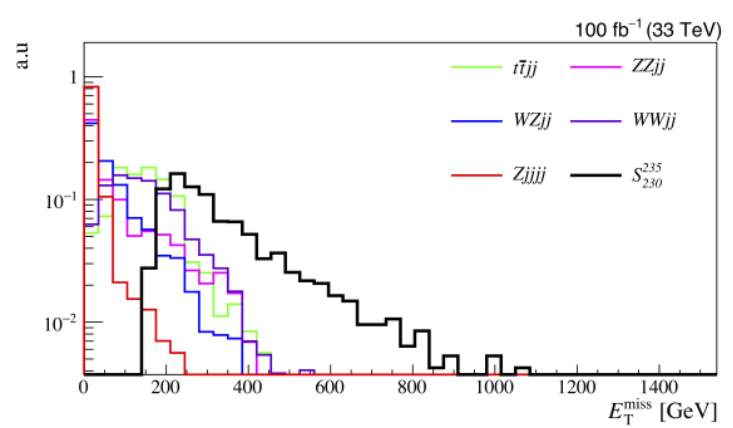

Figure 3. MET distribution, normalized to unity. Events surviving cuts 1-4 are plotted. As seen from the distribution, because of a highly energetic ISR jet requirement, events from signals are mostly populated beyond $180 \mathrm{GeV}$.

Requiring a highly energetic ISR jet will produce a large, anti-collimated missing transverse momentum as well. Hence, in addition to the $180 \mathrm{GeV}$ cut on jet $p_{T}$, we also require a lower cut of $180 \mathrm{GeV}$ on $E_{T}^{\text {miss }}$. As seen from Figure 3 and expected most of the signal events are located beyond $180 \mathrm{GeV}$. A cut of $180 \mathrm{GeV}$ on missing energy increases signal significance $(S / \sqrt{B})$ by a factor of 6 and signal to background ratio by a factor of $\approx 39$.

The transverse momentum of leptons is a measure of the mass difference between slepton and LSP and thus might be a useful variable to reduce the SM backgrounds. Therefore, we imposed an upper cut for the scalar sum of the leptons' transverse momentum. It is shown in Figure 4 , the sum of the leptons' $p_{T}$ for the signal sample separated from SM backgrounds around $80 \mathrm{GeV}$. However, with an upper cut of $50 \mathrm{GeV}$, the highest signal significance is obtained. 
As $\approx 90 \%$ of signal samples and $5 \%$ of $\mathrm{SM}$ backgrounds survive, signal significance and data to $\mathrm{MC}$ ratio increased by a factor of $\approx 3.9$ and $~$ 17 , respectively.

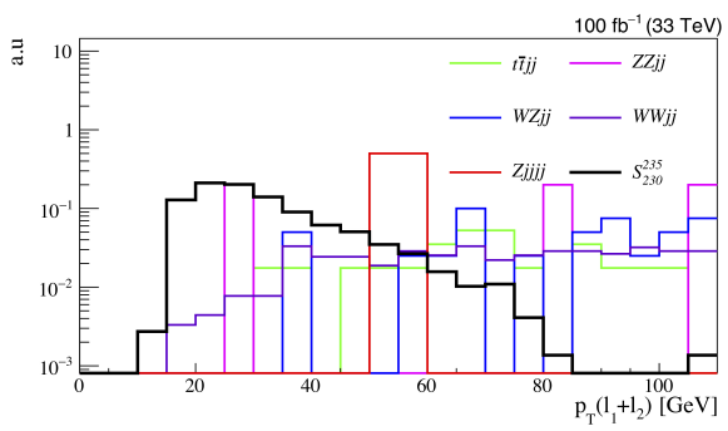

Figure 4. The scalar sum of the transverse momentum of leptons for both signal and background samples. Distribution normalized to unity. Events shown in the plot have passed cuts 1-7. As seen from the distribution, almost no events from signal survive beyond $80 \mathrm{GeV}$, although backgrounds are the most populated ones. An upper cut of 50 $\mathrm{GeV}$ gives the highest significance.

Although upper cut on the scalar sum of leptons' $p_{T}$ helps reduce SM backgrounds, a direct measure of mass difference variable, $\mathrm{m}_{\mathrm{T} 2}-\mu$ is better at separating the backgrounds from the signal. Figure 5 and Figure 6 show $\mathrm{m}_{\mathrm{T} 2}-\mu$ distributions before and after applying an upper cut of $5 \mathrm{GeV}$ on the $\mathrm{m}_{\mathrm{T} 2}-\mu$ variable. As seen from Figure 5, signal events are located mostly below $5 \mathrm{GeV}$, whereas SM backgrounds populated beyond $5 \mathrm{GeV}$. Therefore, applying an upper cut of $5 \mathrm{GeV}$ (for $\Delta \mathrm{M}=3$ $\mathrm{GeV}$ cases, backgrounds and signals are mostly separated with an upper cut of $3 \mathrm{GeV}$ ) dramatically increases the signal background ratio from 0.35 to $\approx$ 3.0 and lets the signal significance reach close to $7.4 \sigma$.

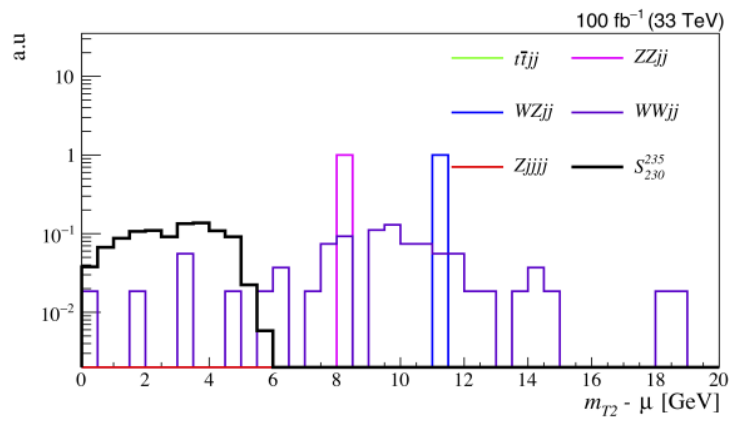

Figure 5. $\mathrm{m}_{\mathrm{T} 2}-\mu$ distribution for backgrounds and signal sample after passing the cuts 1-10. As expected, backgrounds are mostly distributed beyond $5 \mathrm{GeV}$ for the case of $\Delta \mathrm{M}=5 \mathrm{GeV}$

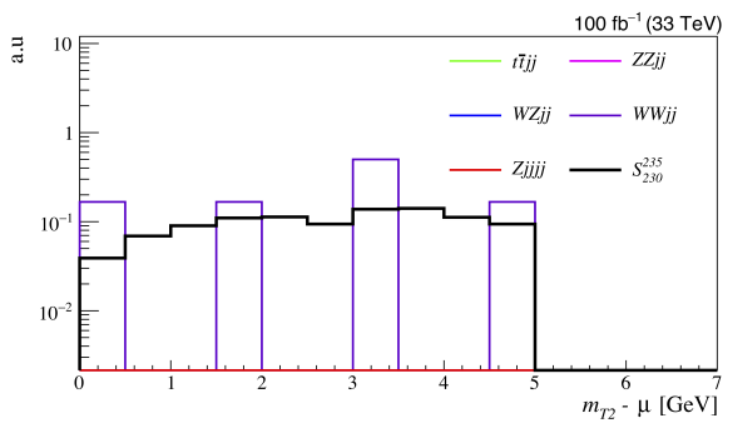

Figure 6. $\mathrm{m}_{\mathrm{T} 2}-\mu$ distribution showing both signal and background events after passing all the cuts 1-11.

\section{CONCLUSION}

Pair production of slepton signal at potential future $p p$ collider colliding protons heads-on at $\sqrt{\mathrm{s}}=33 \mathrm{TeV}$ is probed. The signal events include mass degenerate right/left-handed selectrons and smuons. Branching ratio for the process $\tilde{l} \rightarrow l \widetilde{\chi_{1}^{0}}$ is fixed to $100 \%$. To optimize the kinematic parameters, separating signals from backgrounds, we checked signal significance and signal to background ratio at every single step. With the optimum parameters, we found that, $m_{\tilde{l}} \approx 235 \mathrm{GeV}$ can be reached at the future collider with close to $7.4 \sigma$ discovery in the case where mass difference is $5 \mathrm{GeV}$ (see Figure 7). These selection criteria remain effective for larger masses of choice up to $270 \mathrm{GeV}$ for $\Delta \mathrm{M}=5$ $\mathrm{GeV}$.

Although we assumed $100 \mathrm{fb}^{-1}$ integrated luminosity, a better significance could be obtained with higher luminosity.

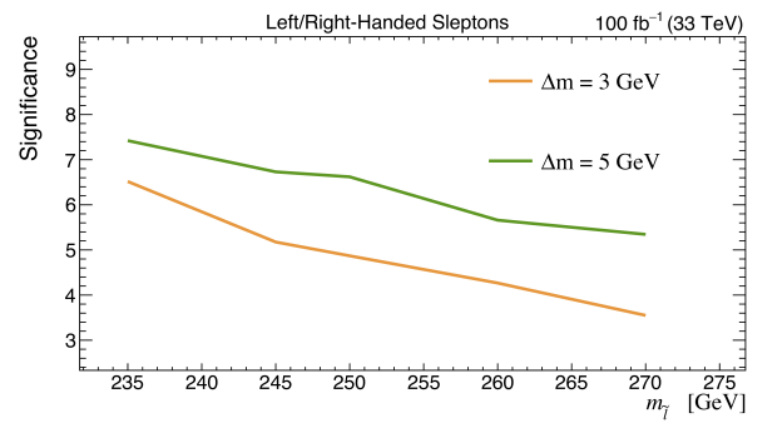

Figure 7. Slepton mass as a function of significance $(S / \sqrt{B})$ for $\Delta M=$ 3 and $5 \mathrm{GeV}$.

\section{Acknowledgement}

This study was supported by Mehmet Akif Ersoy University Scientific Research Projects Unit. Project Number:0669-YL-20. Computing resources used in this work were provided by the National Center for High Performance Computing of Turkey (UHeM) under grant number 1008362020.

\section{REFERENCES}

[1] Ade P. A. R, Aghanim N, Armitage-Caplan C, Arnaud M, Ashdown M, Atrio-Barandele F, et al. Planck2013 results. XVI. Cosmological parameters. Astron Astrophys 2014;571:A16.

[2] Begeman K, Broeils A, Sanders R. Extended rotation curves of spiral galaxies: Dark haloes and modified dynamics. Monthly Notices of the Royal Astronomical Society 1991;249:523-37.

[3] Massey R, Kitching T, Richard J. The dark matter of gravitational lensing. Reports on Progress in Physics 2010;73:086901.

[4] CMS Collaboration. Search for supersymmetry in proton-proton collisions at $13 \mathrm{TeV}$ using identified top quarks. Physical Review D 2018;97:012007.

[5] CMS Collaboration. Search for supersymmetry in multijet events with missing transverse momentum 
in proton-proton collisions at $13 \mathrm{TeV}$. Physical Review D 2017;96:032003.

[6] ATLAS Collaboration. Search for supersymmetry in final states with two same-sign or three leptons and jets using $36 \mathrm{fb}^{-1}$ of $\sqrt{\mathrm{s}}=13 \mathrm{TeV}$ pp collision data with the ATLAS detector. JHEP 2017;09:084.

[7] ATLAS Collaboration. Search for supersymmetry in final states with missing transverse momentum and multiple b-jets in proton-proton collisions at $\sqrt{\mathrm{s}}=13 \mathrm{TeV}$ with the ATLAS detector. JHEP 2018;06:107.

[8] ATLAS Collaboration. Search for squarks and gluinos in final states with jets and missing transverse momentum using $36 \mathrm{fb}^{-1}$ of $\sqrt{\mathrm{s}}=13$ TeV pp collision data with the ATLAS detector. Phys Rev D 2018;97:112001.

[9] CMS Collaboration. Search for Physics Beyond the Standard Model in Events with High-Momentum Higgs Bosons and Missing Transverse Momentum in Proton-Proton Collisions at $13 \mathrm{TeV}$. Phys Rev Lett 2018;120:241801.

[10] CMS Collaboration. Search for new phenomena with the $\mathrm{M}_{\mathrm{T} 2}$ variable in the all-hadronic final state produced in proton-proton collisions at $\sqrt{\mathrm{s}}=13$ TeV. Eur Phys J C 2017;77:710.

[11] ATLAS Collaboration. Search for new phenomena with large jet multiplicities and missing transverse momentum using large-radius jets and flavourtagging at ATLAS in $13 \mathrm{TeV}$ pp collisions. JHEP 2017;12:034.

[12] CMS Collaboration. Search for natural and split supersymmetry in proton-proton collisions at $\sqrt{\mathrm{s}}=13 \mathrm{TeV}$ in final states with jets and missing transverse momentum. Journal of High Energy Physics 2018;2018:1-52.

[13] CMS Collaboration. Searches for electroweak production of charginos, neutralinos, and sleptons decaying to leptons and $\mathrm{W}, \mathrm{Z}$, and Higgs bosons in pp collisions at $8 \mathrm{TeV}$. Eur Phys J C Part Fields 2014;74:3036.

[14] ATLAS Collaboration. Search for direct production of charginos, neutralinos and sleptons in final states with two leptons and missing transverse momentum in pp collisions at $\sqrt{\mathrm{s}}=8 \mathrm{TeV}$ with the ATLAS detector. Journal of High Energy Physics 2014;2014:1-52.

[15] ATLAS Collaboration. Search for electroweak production of supersymmetric states in scenarios with compressed mass spectra at $\sqrt{\mathrm{s}}=13 \mathrm{TeV}$ with the ATLAS detector. Phys Rev D 2018;97:052010.

[16] ATLAS Collaboration. Search for supersymmetric partners of electrons and muons in proton-proton collisions at $\sqrt{\mathrm{s}}=13 \mathrm{TeV}$. Phys Lett B 2019;790:140-66.

https://doi.org/10.1016/j.physletb.2019.01.005.

[17] ATLAS Collaboration. Searches for electroweak production of supersymmetric particles with compressed mass spectra in $\sqrt{\mathrm{s}}=13 \mathrm{TeV} \mathrm{pp}$ collisions with the ATLAS detector. Phys Rev D 2020;101:052005.
[18] Han Z, Liu Y. M ${ }_{\mathrm{T} 2}$ to the Rescue - Searching for Sleptons in Compressed Spectra at the LHC. Phys Rev D 2015;92:015010.

[19] Dutta B, Fantahun K, Fernando A, Ghosh T, Kumar J, Sandick P, et al. Probing Squeezed Bino-Slepton Spectra with the Large Hadron Collider. Phys Rev D 2017;96:075037.

[20] Dutta B, Gosh T, Gurrola A, Johns W, Kamon T, Sheldon P, et.al. Probing compressed sleptons at the LHC using vector boson fusion processes. Phys Rev D 2015;91:055025.

[21] Fayet P. Supersymmetry and weak, electromagnetic and strong interactions. Physics Letters B 1976;64:159-62.

[22] Fayet P. Spontaneously broken supersymmetric theories of weak, electromagnetic and strong interactions. Physics Letters B 1977;69:489-94.

[23] Griest K, Seckel D. Three exceptions in the calculation of relic abundances. Physical Review D 1991;43:3191.

[24] Alwall J, Herquet M, Maltoni F, Mattelaer O, Stelzer T. MadGraph 5: going beyond. J High Energy Phys 2011;2011:1-40.

[25] Sjöstrand T, Mrenna S, Skands P. PYTHIA 6.4 physics and manual. Journal of High Energy Physics 2006;2006:026.

[26] Selvaggi M. DELPHES 3: A modular framework for fast-simulation of generic collider experiments. J. Phys. Conf. Ser., vol. 523, IOP Publishing; 2014, p. 012033.

[27] Selvaggi M [Internet]. HE/HL-LHC detector DELPHES card; 2018 [cited 2021 Nov4]. Available from:

https://github.com/delphes/delphes/blob/9a7ea360f6 f9f1c8f3f35b9545f62c3a61a82230/cards/delphes_c ard_HLLHC.tcl

[28] Mangano ML, others. Matching matrix elements and shower evolution for top-pair production in hadronic collisions. Journal of High Energy Physics 2007;2007:013.

[29] Djouadi A, Muhlleitner M, Spira M. Decays of supersymmetric particles: the program SUSY-HIT (SUspect-SdecaY-HDECAY-InTerface). ArXiv Preprint Hep-Ph/0609292 2006.

[30] Barr AJ. Measuring slepton spin at the LHC. Journal of High Energy Physics 2006;2006:042.

[31] Edelhäuser L, Matchev KT, Park M. Spin effects in the antler event topology at hadron colliders. Journal of High Energy Physics 2012;2012:1-45.

[32] Lester CG, Summers DJ. Measuring masses of semi-invisibly decaying particle pairs produced at hadron colliders. Physics Letters B 1999;463:99_ 103.

[33] Barr A, Lester C, Stephens P. A variable for measuring masses at hadron colliders when missing energy is expected; $\mathrm{M}_{\mathrm{T} 2}$ : the truth behind the glamour. Journal of Physics G: Nuclear and Particle Physics 2003;29:2343-63.

[34] Lester CG, Nachman B. Bisection-based asymmetric $\mathrm{M}_{\mathrm{T} 2}$ computation: a higher precision calculator than existing symmetric methods. J High Energ Phys 2015;2015:100. 